\title{
Fitoterapia no tratamento da candidíase oral: Um protocolo de revisão de escopo
}

\author{
Phytotherapy in the treatment of oral candidiasis: A scoping review protocol \\ Fitoterapia en el tratamiento de la candidiasis oral: Un protocolo de revisión del alcance
}

Recebido: 31/05/2021 | Revisado: 07/06/2021 | Aceito: 10/06/2021 | Publicado: 26/06/2021

\author{
Heloísa Nunes Brandão \\ ORCID: https://orcid.org/0000-0002-9495-1987 \\ Universidade Federal da Paraíba, Brasil \\ E-mail: heloisanb@hotmail.com \\ Ingrid Andrade Meira \\ ORCID: https://orcid.org/0000-0002-3631-0030 \\ Universidade Estadual de Campinas, Brasil \\ E-mail: ingrid_meiraa@hotmail.com \\ Carmem Silvia Laureano Dalle Piagge \\ ORCID: https://orcid.org/0000-0001-7999-2943 \\ Universidade Federal da Paraíba, Brasil \\ E-mail: carmem.piagge@ academico.ufpb.br \\ Dúcia Caldas Cosme-Trindade \\ ORCID: https://orcid.org/0000-0001-9477-7037 \\ Universidade Federal da Paraíba, Brasil \\ E-mail: duciacaldas@hotmail.com
}

\begin{abstract}
Resumo
A Candida albicans é a espécie presente na cavidade oral responsável por cerca de $95 \%$ das candidíases, sendo considerada o principal fator etiológico da estomatite protética, condição inflamatória que acomete a mucosa oral coberta pela base da prótese. O uso de antifúngicos demonstra grande eficácia no tratamento da candidíase oral, apesar de alguns efeitos colaterais, como gosto desagradável, alergias e reações gastrointestinais. Com isso, a fitoterapia vem se destacando como terapia alternativa devido ao seu poder antimicrobiano e a redução de efeitos adversos. Desta forma, o objetivo desta revisão de escopo é explorar sistematicamente a literatura, mapeando e sumarizando as evidências disponíveis quanto aos produtos naturais utilizados no tratamento da candidíase oral. Esse protocolo foi desenvolvido de acordo com as recomendações do Manual do Instituto Joanna Briggs e registrado na Open Science Framework. A busca pelos artigos será realizada em três etapas, utilizando as bases de dados PubMed, Scopus, Web of Science, Embase, Cochrane Library e LILACS, bem como fontes adicionais da literatura cinzenta e lista de referências dos estudos incluídos. Os termos "phytotherapy", "biological products", "oral candidiasis", "denture stomatitis", "candida albicans", e palavras relacionadas serão usadas na estratégia de busca. Os dados serão extraídos através de um formulário e apresentados na forma de quadro e/ou imagens e de forma descritiva. Este protocolo auxiliará o desenvolvimento da revisão de escopo para a identificação de lacunas e síntese do conhecimento para a condução de futuros estudos primários.
\end{abstract}

Palavras-chave: Fitoterapia; Produtos biológicos; Candidíase bucal; Estomatite sob prótese; Candida albicans.

\begin{abstract}
Candida albicans is the microorganism present in the oral mucosa responsible for about $95 \%$ of oral candidiasis. It is considered to be the main etiological factor in denture stomatitis, an inflammation process of the mucosa under the prosthesis. Antifungals are the most predominant treatment against $c$. albicans and demonstrate great efficacy despite many side effects, such as unpleasant taste, allergies and gastrointestinal reactions. Phytotherapy has been showing promise as an alternative therapy due to its advantages, mainly the antimicrobial effect and the reduction of adverse sideeffects. Thus, the objective of this scoping review is to systematically explore the literature, to map and to summarize the available evidence regarding the use of natural products in the treatment of oral candidiasis. This protocol was developed in accordance with the recommendations of the Joanna Briggs Institute Manual and registered with Open Science Framework. The search for the articles will be carried out in three stages, using the PubMed, Scopus, Web of Science, Embase, Cochrane Library and LILACS databases, as well as additional sources of gray literature and reference lists of included studies. The terms "phytotherapy", "biological products", "oral candidiasis", "denture stomatitis", "candida albicans", and their related words will be used in this strategy. The data will be extracted into a form and presented as tables and/or images and as a narrative. This protocol will assist in the development of the scoping review to identify gaps and synthesize knowledge for the conduct of future primary studies.
\end{abstract}

Keywords: Phytotherapy; Biological products; Candidiasis, Oral; Stomatitis, Denture; Candida albicans. 


\begin{abstract}
Resumen
Candida albicans es la especie presente en la cavidad bucal responsable de alrededor del $95 \%$ de las candidiasis, siendo considerada el principal factor etiológico de la estomatitis subprotética, una condición inflamatoria que afecta a la mucosa bucal recubierta por la base de la prótesis. El uso de antifúngicos muestra una gran eficacia en el tratamiento de la candidiasis, a pesar de algunos efectos secundarios, como sabor desagradable, alergias y reacciones gastrointestinales. La fitoterapia se ha destacado como una terapia alternativa por su poder antimicrobiano y la reducción de efectos adversos. El propósito de esta revisión del alcance es explorar sistemáticamente la literatura, mapear y resumir la evidencia disponible sobre los productos naturales utilizados en el tratamiento de la candidiasis oral. Este protocolo fue desarrollado de acuerdo con las recomendaciones del Manual del Instituto Joanna Briggs y registrado en el Open Science Framework. La búsqueda de los artículos se realizará en tres etapas, utilizando las bases de datos PubMed, Scopus, Web of Science, Embase, Cochrane Library y LILACS, así como fuentes adicionales de literatura gris y lista de referencias de los estudios incluidos. Los términos "fitoterapia", "productos biológicos", "candidiasis oral", "estomatitis subprotética", "candida albicans" y palabras relacionadas se utilizarán en la estrategia de búsqueda. Los datos se extraerán a través de un formulario y se presentarán en forma de tablas y/o imágenes y en forma narrativa. Este protocolo ayudará en el desarrollo de la revisión del alcance para la identificación de lagunas y la síntesis de conocimientos.

Palabras clave: Fitoterapia; Productos biológicos; Candidiasis bucal; Estomatitis subprotética; Candida albicans.
\end{abstract}

\title{
1. Introdução
}

A Candida spp. é um fungo comensal da microbiota oral saudável e que pode se tornar patogênico em condições que favoreçam a sua multiplicação (Muadcheingka \& Tantivitayakul, 2015; Lewis \& Williams, 2017), como reduzido fluxo salivar, deficiência vitamínica, fumo, terapias com antibióticos de largo espectro e presença de doenças imunossupressoras (Hu et al., 2019; Vila, Sultan, Montelongo-Jauregui \& Jabra-Rizk, 2020). A Candida albicans é a espécie mais presente na cavidade bucal e responsável por cerca de 95\% das candidíases (Vila et al., 2020), sendo também comumente associada à estomatite protética, condição caracterizada pela inflamação dos tecidos cobertos pelas bases das próteses (Yarborough et al., 2016; Hannah, O'Donnell, Robertson \& Ramage, 2017). As alterações decorrentes do processo de envelhecimento, além do uso de diversas medicações, favorecerem a instalação da candidíase oral nos indivíduos idosos (Hu et al., 2019; Vila et al., 2020; Buranarom, Komin \& Matangkasombut, 2020). Além disso, a precária higiene e qualidade das próteses, assim como o uso constante delas, contribuem no acúmulo do biofilme e no desenvolvimento e/ou perpetuação da inflamação (Hannah et al., 2017; Vila et al., 2020).

Os idosos representavam, em 2013, $13 \%$ da população brasileira (IBGE, 2013), com projeções de que em 2040 este número duplique (IBGE, 2018). O edentulismo nestes indivíduos ainda é uma realidade, com 63,1\% sendo desdentados totais na maxila e 15,4\% necessitando de prótese total dupla (SB Brasil 2010), com projeções de que em 2040 será alcançado o número de 64 milhões de idosos edêntulos (Cardoso, Balducci, Telles, Lourenço \& Júnior, 2016). Desta forma, diante da prevalência da candidíase em usuários de próteses, torna-se fundamental a busca por evidências no tratamento desta condição, que é também uma das lesões de mucosa oral mais prevalentes nos idosos (Peres, Barbato, Reis, Freitas \& Antunes, 2013; Lynge Pedersen, Nauntofte, Smidt \& Torpet, 2015; Azevedo, Azevedo, Oliveira, Correa, \& Demarco, 2017). Isto porque a existência de um ambiente favorável à propagação fúngica (presença da prótese), também pode ser um fator de risco para doenças sistêmicas (Le Bars, Kouadio, N'goran, Badran \& Soueidan, 2015), principalmente em idosos mais debilitados, que utilizam várias medicações simultaneamente (Gacon \& Wieczorek, 2020; Buranarom et al., 2020).

O tratamento da candidíase oral com antifúngicos apresenta grande eficácia, porém seu uso pode estar associado a reações adversas, como alterações no paladar, reações gastrointestinais e alergias (Bakhshi, Taheri, Shabestari, Tanik, \& Pahlevan, 2012). Estudos recentes têm revelado a resistência da Candida spp. aos antifúngicos devido ao seu uso frequente e duradouro (Maubon, Garnaud, Calandra, Sanglard \& Cornet, 2014; Bailly et al., 2016), fato comum em usuários de próteses (Bailly et al., 2016; Lewis \& William, 2017; Gad \& Fouda, 2020). A terapia fotodinâmica, a desinfecção por microoondas, o uso de soluções desinfetantes e o uso de produtos fitoterápicos têm sido sugeridos como alternativas aos antifúngicos (Emami, Kabawat, Rompre \& Feine, 2014). Dentre estes, a fitoterapia tem se destacado devido à sua ação antimicrobiana e antiinflamatória, reações tóxicas 
diminuídas, redução no desenvolvimento de cepas resistentes, alta biocompatibilidade e baixo custo, sendo considerada uma alternativa promissora no tratamento da candidíase oral e/ou estomatite protética (Ferreira et al., 2015; Gad \& Fouda, 2020; Shui, Li, Lyu \& Wang, 2021).

Embora a literatura aponte diferentes produtos naturais para o tratamento da candidíase oral, nenhuma revisão de escopo foi encontrada nas bases de dados, nem nas plataformas de registro Open Science Framework e Figshare, com o objetivo de explorar o que há na literatura sobre o uso destes produtos no manejo do paciente com infecção por Candida albicans. Uma revisão sistemática foi realizada há mais de seis anos (Ferreira et al., 2015), porém os autores encontraram grande heterogeneidade quanto ao delineamento dos estudos e aos produtos e posologias utilizados, demonstrando a ausência de evidência científica no tratamento da candidíase oral. Uma outra revisão sistemática (Shui et al., 2021) sobre a eficácia dos fitoterápicos no tratamento da estomatite protética concluiu que, embora o uso seja promissor, devido aos baixos efeitos adversos, são necessários mais ensaios clínicos randomizados para confirmar a sua eficácia como substitutos dos antifúngicos.

Dessa forma, o objetivo desta revisão de escopo é explorar sistematicamente a literatura quanto aos produtos naturais mais utilizados no tratamento da candidíase oral, mapeando e sumarizando as evidências quanto às posologias e formas de apresentação mais adotadas, tipos de respostas quando comparados aos tratamentos tradicionais, verificando como os estudos vêm sendo conduzidos e identificando lacunas na literatura. Com isso, essa revisão sintetizará o conhecimento existente para a condução de futuros estudos primários, determinando a possibilidade de execução de uma revisão sistemática sobre o tópico mais adiante.

\subsection{Pergunta de Pesquisa}

A seguinte pergunta de pesquisa incorpora os elementos do PCC (Participantes, Conceito, Contexto), orientando e direcionando na definição dos critérios de inclusão específicos para esta revisão:

- Quais são as evidências científicas do uso da fitoterapia no tratamento da candidíase oral?

\subsection{Critérios de inclusão}

\subsubsection{Participantes}

Estudos clínicos conduzidos em pacientes diagnosticados com candidíase oral e/ou estomatite protética, sem distinção de sexo, idade ou etnia, serão incluídos nesta revisão. Além disso, estudos laboratoriais (in vitro e com modelo animal) também relacionados à candidíase oral serão incluídos.

\subsubsection{Conceito}

O conceito de interesse para esta revisão é a existência de evidência científica que suporte o uso da fitoterapia no tratamento da candidíase oral. Portanto, estudos que analisaram qualquer produto natural, independentemente do modo de apresentação e da posologia adotados, serão considerados nesta revisão de escopo.

\subsubsection{Contexto}

O contexto desta revisão é considerado aberto já que fontes de evidência pertencentes a qualquer configuração contextual serão elegíveis para inclusão. Nenhum contexto específico será determinado para refinar o escopo da revisão.

\subsubsection{Tipos de fontes de evidência}

Nesta revisão de escopo serão considerados todos os estudos experimentais e quase-experimentais, além de estudos observacionais analíticos, incluindo estudos de coorte prospectivos e retrospectivos, estudos de caso-controle e estudos 
transversais. Também serão considerados estudos observacionais descritivos, como séries de casos e relatos de casos, pesquisas qualitativas, estudos secundários e diretrizes de prática clínica. Além disso, fontes adicionais provenientes da literatura cinzenta como teses, textos e artigos de opinião também serão incluídos.

\section{Metodologia}

Essa revisão de escopo será conduzida de acordo com as recomendações de estruturação do Manual do Instituto Joanna Briggs - JBI Manual for Evidence Synthesis (Peters, Godfrey, McInerney, Munn, Tricco \& Khalil, 2020), tendo sido registrada na Open Science Framework (https://osf.io/) com o número DOI 10.17605/OSF.IO/N32VG (osf.io/8bxud).

\subsection{Estratégia de busca}

A estratégia será elaborada de modo a identificar as possíveis fontes de evidências publicadas nas diversas bases de dados, bem como na literatura cinzenta. Buscando uma maior abrangência na captura dos estudos, será realizada uma busca inicial nas bases PubMed e Web of Science por meio da testagem de termos Mesh e termos índices, analisando títulos, resumos e palavraschaves dos artigos recuperados para identificação de possíveis termos que possam ser adicionados à estratégia de busca. Após essa primeira etapa, a estratégia de busca para o PubMed será concluída e, em seguida, adaptada para as demais bases de dados a serem utilizadas na revisão, de acordo com as particularidades de cada uma. A estratégia de busca para o PubMed está apresentada no Quadro 1. Na terceira e última etapa da busca, as listas de referências dos artigos incluídos nesta revisão de escopo serão examinadas, buscando a identificação de algum estudo adicional.

Quadro 1. Estratégia de busca preliminar para o PubMed.

\begin{tabular}{|l|l|l|}
\hline $\begin{array}{l}\text { Base de } \\
\text { dados }\end{array}$ & \multicolumn{1}{|c|}{ Estratégia de busca } & $\begin{array}{c}\text { Resultados } \\
18 / 05 / 2021\end{array}$ \\
\hline \multirow{5}{*}{ PubMed } & $\begin{array}{l}\text { ("Phytotherapy"[MeSH Terms] OR "Phytotherapy"[Title/Abstract] OR "Herbal } \\
\text { Therapy"[Title/Abstract] OR "Herb Therapy"[Title/Abstract] OR } \\
\text { "phytotherapeutic"[Title/Abstract] OR "phytotherapic"[Title/Abstract] OR "Biological } \\
\text { Products"[MeSH Terms:noexp] OR "Biological Product"[Title/Abstract] OR "Biologic } \\
\text { Products"[Title/Abstract] OR "Biological Drug"[Title/Abstract] OR "Biologic } \\
\text { Drugs"[Title/Abstract] OR "Biological Medicines"[Title/Abstract] OR "Biologic } \\
\text { Medicines"[Title/Abstract] OR "Biological Drugs"[Title/Abstract] OR "Natural } \\
\text { Products"[Title/Abstract] OR "Natural Product"[Title/Abstract] OR "herbal } \\
\text { remedies"[Title/Abstract] OR "herbal medicines"[Title/Abstract] OR "Biological } \\
\text { Medicine"[Title/Abstract] OR "Biologic Drug"[Title/Abstract]) AND ("candidiasis, oral"[MeSH } \\
\text { Terms] OR "Oral Candidiasis"[Title/Abstract] OR "Thrush"[Title/Abstract] OR "Oral } \\
\text { Moniliasis"[Title/Abstract] OR "stomatitis, denture"[MeSH Terms] OR "Denture } \\
\text { stomatitis"[Title/Abstract] OR "Candida albicans"[MeSH Terms] OR "Candida } \\
\text { albicans"[Title/Abstract]) }\end{array}$ \\
\hline
\end{tabular}

Fonte: Autores.

Uma bibliotecária experiente supervisionará todo o processo de montagem da estratégia de busca e refinamento. Não haverá nenhuma restrição temporal e de idioma na busca das evidências, bem como caso sejam necessárias mais informações, o autor do estudo primário será contatado. Durante o desenvolvimento da revisão, caso os revisores identifiquem palavras-chaves, termos livres ou fontes adicionais de interesse, estas serão incorporadas à estratégia de busca, e informadas com transparência na versão final da revisão.

\subsection{Fontes de informações}


Além do PubMed, as seguintes bases de dados serão utilizadas para identificar os estudos relevantes: Scopus, Web of Science, Embase, Cochrane Library e LILACS. A busca da literatura cinzenta será realizada por meio do Google Scholar, OpenGrey e ProQuest Dissertations \& Theses Global.

\subsection{Seleção dos estudos}

Após as buscas nas bases de dados indicadas, todos os registros serão exportados para o gerenciador de referências EndNote (Clarivate Analytics, PA, USA), onde serão agrupados para a remoção automática dos artigos duplicados. Em seguida, os artigos serão exportados para o Software Rayaan (Qatar Computing Research Institute) (Ouzzani et al., 2016), onde será conduzida a fase de seleção dos estudos em duas etapas: na fase 1 os artigos serão selecionados com base na análise do título e leitura do resumo; na fase 2 será realizada a leitura completa dos artigos incluídos, sempre levando em consideração os critérios de inclusão pré-estabelecidos no protocolo. Nas duas fases, dois revisores independentes realizarão a triagem (leitura de título e resumo) e a leitura dos textos completos, e qualquer divergência entre eles será resolvida por consenso ou pela decisão do terceiro revisor. Seguindo as recomendações do Manual do JBI, um teste piloto para a seleção dos artigos será realizado previamente, com análise e discussão dos critérios de inclusão, buscando uma concordância de no mínimo 75\% entre os revisores.

Todo este processo será reportado na versão final da revisão de escopo, apresentado na forma de um fluxograma, detalhando os resultados das fontes de evidência (bases de dados e fontes adicionais), remoção de duplicatas, triagem e seleção dos estudos na fase 1 e 2, com as razões pelas quais foram excluídos, e o número de estudos incluídos na revisão. As diretrizes do Preferred Reporting Items for Systematic Reviews and Meta-Analyses extension for Scoping Reviews (PRISMA-ScR) Checklist serão adotadas na elaboração do artigo desta revisão de escopo (Tricco et al., 2018).

\subsection{Extração de Dados}

A extração de dados dos estudos incluídos na revisão será realizada de forma independente pelos dois revisores, por meio de um formulário desenvolvido pelos próprios autores, seguindo a sugestão do Manual do JBI. Os dados incluirão informaçõeschave da fonte de evidência como autor, ano de publicação, país, objetivo do estudo, tipo de estudo (clínico ou laboratorial) /delineamento, população e tamanho da amostra, metodologia, nome do fitoterápico, posologia e formas de apresentação adotadas, comparador, principais resultados ou descobertas relacionadas à pergunta de pesquisa, e comentários. Um teste piloto com três artigos será realizado com o objetivo de aumentar a concordância entre os revisores. Qualquer modificação no formulário poderá ser realizada e será relatada na versão final da revisão. Assim como na fase de seleção dos estudos, as divergências entre os revisores serão resolvidas em consenso com um terceiro revisor.

\subsection{Apresentação dos dados}

Os dados extraídos serão apresentados na forma de tabelas e quadros e/ou imagens, alinhados com o objetivo dessa revisão de escopo. Além disso, os dados resultantes da extração serão sintetizados e descritos de uma forma narrativa, com o objetivo de facilitar a compreensão do leitor quanto ao tópico de pesquisa.

\section{Conflitos de interesse}

Os autores declaram que não há conflito de interesse.

\section{Referências}


Azevedo, J. S., Azevedo, M. S., Oliveira, L. J. C. de, Correa, M. B., \& Demarco, F. F. (2017). Uso e necessidade de prótese dentária em idosos brasileiros segundo a Pesquisa Nacional de Saúde Bucal (SBBrasil 2010): prevalências e fatores associados. Cadernos de Saúde Pública, 33(8), e00054016. Epub August 21, 2017.

Bailly, S., Maubon, D., Fournier, P., Pelloux, H., Schwebel, C., Chapuis, C., Foroni, L., Cornet, M. \& Timsit, J. F. (2016). Impact of antifungal prescription on relative distribution and susceptibility of Candida spp. - Trends over 10 years. The Journal of infection, 72(1), 103-111.

Bakhshi, M., Taheri, J. B., Shabestari, S. B., Tanik, A. \& Pahlevan, R. (2012). Comparison of therapeutic effect of aqueous extract of garlic and nystatin mouthwash in denture stomatitis. Gerodontology, 29(2), e680-e684.

Brasil. Ministério da Saúde. Secretaria de Atenção à Saúde. Secretaria de Vigilância em Saúde. SB Brasil 2010: Pesquisa Nacional de Saúde Bucal: resultados principais / Ministério da Saúde. Secretaria de Atenção à Saúde. Secretaria de Vigilância em Saúde. - Brasília: Ministério da Saúde, 2012. 116 p. ISBN 978-85334-1987-2

Buranarom, N., Komin, O., \& Matangkasombut, O. (2020). Hyposalivation, oral health, and Candida colonization in independent dentate elders. PloS one, 15(11), e0242832.

Cardoso, M., Balducci, I., Telles, D. M., Lourenço, E. J. V. \& Júnior, L. N. (2016). Edentulism in Brazil: trends, projections and expectations until 2040. Ciência \& Saúde Coletiva, 21(4), 1239-46.

Emami, E., Kabawat, M., Rompre, P. H. \& Feine, J. S. (2014). Linking evidence to treatment for denture stomatitis: a meta-analysis of randomized controlled trials. Journal of dentistry, 42(2), 99-106.

Ferreira, G. L., Pérez, A. L., Rocha, Í. M., Pinheiro, M. A., de Castro, R. D., Carlo, H. L., Lima, E. \& Castellano, L. R. (2015). Does scientific evidence for the use of natural products in the treatment of oral candidiasis exist? A systematic review. Evidence-based complementary and alternative medicine: eCAM, 2015, 147804.

Gacon, I. \& Wieczorek, A. (2020). Coexistence of Lack of Clinical Manifestation of Oral Mycosis and Systemic Diseases in Edentulous Patients Using Removable Prosthetic Restorations. International journal of environmental research and public health, 17(17), 6348.

Gad, M. M. \& Fouda, S. M. (2020). Current perspectives and the future of Candida albicans-associated denture stomatitis treatment. Dental and medical problems, 57(1), 95-102.

Hannah, V. E., O'Donnell, L., Robertson, D. \& Ramage, G. (2017). Denture Stomatitis: Causes, Cures and Prevention. Primary dental journal, 6(4), 46-51.

Hu, L., He, C., Zhao, C., Chen, X., Hua, H. \& Yan, Z. (2019). Characterization of oral candidiasis and the Candida species profile in patients with oral mucosal diseases. Microbial pathogenesis, 134, 103575.

Instituto Brasileiro de Geografia e Estatística, IBGE (2015). Pesquisa Nacional por Amostras de Domicílio: síntese de indicadores, 2013. https://biblioteca.ibge.gov.br/visualizacao/livros/liv94414.pdf

Instituto Brasileiro de Geografia e Estatística, IBGE (2018). Projeções da população. https://www.ibge.gov.br/estatisticas-novoportal/sociais/populacao/9109projecao-da-populacao.html?=\&t=downloads.

Le Bars, P., Kouadio, A. A., N'goran, J. K., Badran, Z. \& Soueidan, A. (2015). Relationship between removable prosthesis and some systemics disorders. Journal of Indian Prosthodontic Society, 15(4), 292-299.

Lewis, M. \& Williams, D. W. (2017). Diagnosis and management of oral candidosis. British dental journal, $223(9), 675-681$.

Lynge Pedersen, A. M., Nauntofte, B., Smidt, D. \& Torpet, L. A. (2015). Oral mucosal lesions in older people: relation to salivary secretion, systemic diseases and medications. Oral diseases, 21(6), 721-729.

Maubon, D., Garnaud, C., Calandra, T., Sanglard, D. \& Cornet, M. (2014). Resistance of Candida spp. to antifungal drugs in the ICU: where are we now? Intensive care medicine, 40(9), 1241-1255.

Muadcheingka, T. \& Tantivitayakul, P. (2015). Distribution of Candida albicans and non-albicans Candida species in oral candidiasis patients: Correlation between cell surface hydrophobicity and biofilm forming activities. Archives of oral biology, 60(6), 894-901.

Ouzzani, M., Hammady, H., Fedorowicz, Z. \& Elmagarmid, A. Rayyan-a web and mobile app for systematic reviews. Systematic Reviews. 2016; 5:1-10.

Tricco, A.C., Lillie, E., Zarin, W., O'Brien, K.K., Colquhoun, H., Levac, D., Moher, D., Peters, M.D., Horsley, T., Weeks, L. \& Hempel, S., (2018). PRISMA extension for scoping reviews (PRISMA-ScR): checklist and explanation. Annals of internal medicine, 169(7), 467-473.

Peres, M. A., Barbato, P. R., Reis, S. C. G. B., Freitas, C. H. S. de M. \& Antunes, J. L. F. (2013). Perdas dentárias no Brasil: análise da Pesquisa Nacional de Saúde Bucal 2010. Revista de Saúde Pública, 47(Suppl. 3), 78-89.

Peters, M.D.J., Godfrey, C., McInerney, P., Munn, Z., Tricco, A.C., Khalil, H. Chapter 11: Scoping Reviews (2020 version). In: Aromataris E, Munn Z (Editors). JBI Manual for Evidence Synthesis, JBI, 2020. https://synthesismanual.jbi.global. https://doi.org/10.46658/JBIMES-20-12

Shui, Y., Li, J., Lyu, X. \& Wang, Y. (2021). Phytotherapy in the management of denture stomatitis: A systematic review and meta-analysis of randomized controlled trials. Phytotherapy Research, 1- 16.

Vila, T., Sultan, A. S., Montelongo-Jauregui, D. \& Jabra-Rizk, M. A. (2020). Oral Candidiasis: A Disease of Opportunity. Journal of fungi (Basel, Switzerland), $6(1), 15$.

Yarborough, A., Cooper, L., Duqum, I., Mendonça, G., McGraw, K. \& Stoner, L. (2016). Evidence Regarding the Treatment of Denture Stomatitis. Journal of prosthodontics: official journal of the American College of Prosthodontists, 25(4), 288-301. 
Research, Society and Development, v. 10, n. 7, e35010716653, 2021

(CC BY 4.0) | ISSN 2525-3409 | DOI: http://dx.doi.org/10.33448/rsd-v10i7.16653 